\title{
Energy Efficient and Secure Multipoint Relay Selection in Mobile Ad hoc Networks
}

\author{
Anjali Anand $^{1}$, Rinkle Rani ${ }^{2}$ and Himanshu Aggarwal ${ }^{1}$ \\ ${ }^{1}$ Department of Computer Engineering, Punjabi University, \\ Patiala-147002, India \\ [e-mail: anjalianand_87@yahoo.in, himanshu.pup@gmail.com] \\ ${ }^{2}$ Computer Science and Engineering Department, Thapar University, \\ Patiala-147004, India \\ [e-mail: raggarwal@thapar.edu] \\ *Corresponding author: Anjali Anand \\ Received October 22, 2015; revised January 5, 2016; accepted February 2, 2016; \\ published April 30, 2016
}

\begin{abstract}
Nodes in MANETs are battery powered which makes energy an invaluable resource. In OLSR, MPRs are special nodes that are selected by other nodes to relay their data/control traffic which may lead to high energy consumption of MPR nodes. Therefore, employing energy efficient MPR selection mechanism is imperative to ensure prolonged network lifetime. However, misbehaving MPR nodes tend to preserve their energy by dropping packets of other nodes instead of forwarding them. This leads to huge energy loss and performance degradation of existing energy efficient MPR selection schemes. This paper proposes an energy efficient secure MPR selection (ES-MPR) technique that takes into account both energy and security metrics for MPR selection. It introduces the concept of 'Composite Eligibility Index' (CEI) to examine the eligibility of a node for being selected as an MPR. CEI is used in conjunction with willingness to provide distinct selection parameters for Flooding and Routing MPRs. Simulation studies reveal the efficiency of ES-MPR in selection of energy efficient secure and stable MPRs, in turn, prolonging the network operational lifetime.
\end{abstract}

Keywords: energy efficient, MPR selection, OLSR, misbehaving node, MANET security, optimized link state routing. 


\section{Introduction}

Mobile ad hoc networks (MANETs) comprise of mobile stations or nodes autonomously configured in an infratructureless environment. The ability to operate rapidly without any centralized authority makes MANET a suitable candidate for various applications ranging from military and rescue operations to mobile conferencing, environmental monitoring etc. Dynamic topology due to mobility of nodes and lack of central administrating authority makes routing a challenging task in such networks. Various routing protocols have been proposed by Internet Engineering Task Force (IETF) such as Optimized Link State Routing (OLSR) protocol, Ad hoc On-demand Distance Vector (AODV) protocol and Dymanic Source Routing (DSR) protocol for MANETs. The details about these protocols have been given in [1] [2].

OLSR [3] is a popular proactive routing protocol in which nodes maintain paths to all destinations in the network. This is done by performing periodic exchange of control messages (HELLO and TC messages) among nodes in the network. In OLSR, specific nodes are selected for the purpose of broadcasting information known as Multipoint Relay (MPR) nodes. Selecting subset of one-hop neighbours as MPRs ensures reduction of redundant information in the network, thereby reducing collisions and energy consumption of nodes. OLSRv2 [4] is an updation of OLSR which provides two kinds of MPR nodes, namely Routing MPRs and Flooding MPRs in order to achieve topology reduction and flooding reduction respectively. Routing MPRs are responsible for relaying data packets for the selector node, whereas Flooding MPRs are used to spread topology control information in the network. Fig. 1 shows different Flooding and Routing MPRs selected by the source node.

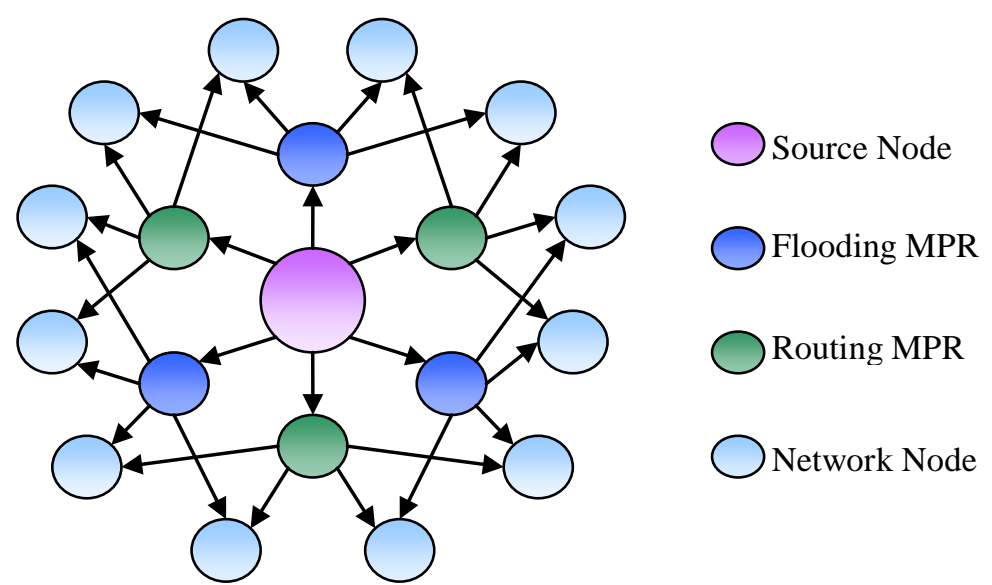

Fig. 1. Flooding and routing MPRs

Energy is a crucial parameter that must be taken into account while devising a mechanism for MPR selection due to two major factors: Firstly, energy consumption of MPR nodes is higher than non-MPR nodes as they have to relay data/control traffic on behalf of the nodes 
(MPR selector) that have selected them as MPR. Secondly, battery powered nodes in MANETs have limited energy resource available with them. Furthermore, due to paucity of energy and high energy consumption, nodes tend to misbehave in order to conserve their energy by dropping packets of the sender node in guise of forwarding them. This results in energy loss at the sender node. Therefore, this paper proposes an energy efficient secure MPR selection mechanism, i.e. ES-MPR that considers both energy metrics and presence of misbehaviour in the network for MPR selection.

Various researchers have worked in the direction of providing MPR selection mechanism for OLSR protocol[5][6][7][8][9][10][11][12][13][14][15][16][17][18][19][20][21].

EE-OLSR [5] provides an energy aware willingness computation mechanism based on the battery capacity and lifetime of nodes. The willingness of nodes take a value out of the three (Default, Low, High) depending upon the battery, lifetime pair.

REOLSR2 [6] modifies the standard MPR selection mechanism in OLSR protocol by taking into account residual energy, reachability and degree of one-hop neighbours to avoid high energy consumption of specific nodes in the network.

RBC-OLSR [7] is a reputation based scheme for selection of trusted paths. It aims to provide reduction in the number of relay nodes and considers residual energy along with connectivity of nodes for MPR selection.

In paper [8], the authors have proposed a fuzzy based MPR selection technique designed to work in high mobility and high loss rate environment. It provides an improved OLSR by integrating various factors such as mobility, distance and received signal strength in MPR selection mechanism.

FQOLSR [9] provides a fuzzy based MPR selection mechanism for OLSR protocol using Mamdani Inference methodology. It provides an algorithm for selection of quality MPRs (QMPR) for QoS support in OLSR. It predicts quality of nodes for MPR selection using a composite metric based on energy, stability and buffer occupancy.

In paper [10], the authors have proposed two mechanisms, namely Stability and Fidelity of nodes for improvement of MPR selection algorithm. Stability of nodes is determined using Bicnayme-Chebyshev inequality and received signal power at different time intervals. On the other hand, Fidelity of nodes is defined as the reachabiliy of nodes. A combination of Stability and Fidelity of nodes are used for election of stable MPR nodes and maintaing stable network.

In paper [11], the authors aim to provide stable and durable paths for communication in the network. MPR selection is based on three criteria: residual energy, link stability and queue capacity of the nodes. This scheme is referred to as ELSQ.

MBQA-OLSR [12] is a multipath energy efficient protocol which is an improvement of MP-OLSR. It proposes energy aware MPR selection including a willingness setting mechanism based on residual energy, lifetime and idle time of the nodes in the network. MPR nodes are selected by MPR selector based upon willingness, degree and reachability of one-hop neighbours.

The above mentioned MPR selection schemes provide willingness computation 
mechanisms based on energy and other QoS parameters but do not take into account misbehaviour of nodes in the network and may fail to perform in hostile environment. The proposed scheme ES-MPR considers both energy metrics and presence of misbehaviour in the network for MPR selection. Unlike existing schemes, ES-MPR provides distinct selection parameters for selection of Routing and Flooding MPRs. It introduces the concept of 'Composite Eligibility Index' which is employed along with willingness for determining how eligible a node is to be selected as an MPR. Table 1 shows the comparison of ES-MPR with the existing MPR selection schemes.

Table 1. Comparison of ES-MPR with existing MPR selection schemes

\begin{tabular}{|l|c|c|c|c|c|c|}
\hline \multicolumn{1}{|c|}{ FEATURES } & $\begin{array}{c}\text { EE-OLSR } \\
{[5]}\end{array}$ & $\begin{array}{c}\text { REOLSR2 } \\
{[6]}\end{array}$ & $\begin{array}{c}\text { FQOLSR } \\
{[9]}\end{array}$ & $\begin{array}{c}\text { ELSQ } \\
{[11]}\end{array}$ & $\begin{array}{c}\text { MBQA- } \\
\text { OLSR } \\
{[12]}\end{array}$ & ES-MPR \\
\hline Residual Energy & Yes & Yes & Yes & Yes & Yes & Yes \\
\hline Drain Rate & Yes & No & No & No & Yes & Yes \\
\hline Available Bandwidth & No & No & No & No & No & Yes \\
\hline Queue Occupancy & No & No & Yes & Yes & Yes & Yes \\
\hline HELLO Loss Ratio & No & No & No & No & No & Yes \\
\hline Transmission Power & No & No & No & No & No & Yes \\
\hline Signal Stability & No & No & Yes & Yes & No & Yes \\
\hline Incentive Mechanism & No & No & No & No & No & Yes \\
\hline
\end{tabular}

The next section illustrates the impact of misbehaviour on energy consumption. Section 3 presents the details of the proposed scheme. Section 4 entails the simulation environment and performance evaluation of the proposed scheme. The last section includes the conclusion of the proposed work.

\section{Impact of Misbehaviour on Energy Consumption}

This section analyzes the effect of misbehaving MPR node on energy consumption of MPR selector node based upon misbehaving probability of MPR node $P_{M}$, which is defined as the number of packets dropped over the total number of packets. For determining the energy consumption due to misbehaviour, Expected Transmission Count (ETX) [22] of MPR selector node is computed. It represents the number of times a selector node has to transmit the packet to MPR node in order to ensure its successful forwarding by the corresponding MPR node.

This analysis is based on the following assumptions:

- Misbehaving probability of nodes is independent of each other.

- Nodes are uniformly distributed over the network area.

- Source and destination pairs are randomly selected. 
The analysis includes computation of Expected Transmission Count (ETX) of selector node based upon misbehaving probability $P_{M}$, of the MPR node.

$$
\operatorname{ETX}_{P M}(M P R)=\frac{1}{1-P_{M}}
$$

Let $\varepsilon$ be the energy consumption of MPR selector node assuming that the MPR node is not misbehaving. Then, energy consumption of selector node for successful transmission of a packet through an MPR node having misbehaving probability $P_{M}$, is represented as:

$$
\begin{aligned}
\varepsilon_{P M} & =E T X_{P M}(M P R)^{*} \varepsilon \\
& =\frac{1}{1-P_{M}} * \varepsilon
\end{aligned}
$$

Table 2 illustrates $\varepsilon_{P M}$ for various misbehaving probabilities of MPR node.

Table 2. $\varepsilon_{P M}$ for varying misbehaving probability of nodes

\begin{tabular}{|c|c|c|}
\hline$P_{M}$ & $E T X_{P M}(M P R)$ & $\varepsilon_{P M}$ \\
\hline 0.1 & 1.1 & $1.1 \varepsilon$ \\
\hline 0.2 & 1.25 & $1.25 \varepsilon$ \\
\hline 0.3 & 1.42 & $1.42 \varepsilon$ \\
\hline 0.4 & 1.66 & $1.66 \varepsilon$ \\
\hline 0.5 & 2 & $2 \varepsilon$ \\
\hline
\end{tabular}

From Table 2, it can be observed the energy consumption of MPR selector node for successful transmission of a packet through MPR node increases with increasing misbehaving probability $P_{M}$ of MPR node. For example, when MPR node with $P_{M}=0.3$ is selected, then the energy consumption of the selector node to transmit a packet successfully through the corresponding MPR node is almost 1.5 times than the energy consumed if MPR node is not misbehaving. Similarly, when $P_{M}=0.5$, the energy consumption is doubled, i.e. $2 \varepsilon$. This can lead to increased battery consumption of nodes in the network and shortens network operational lifetime. Hence, it becomes indispensable to devise energy efficient and secure MPR selection mechanism for prolonging network lifetime which is the premise of this research work.

\section{Energy Efficient and Secure MPR Selection (ES-MPR)}

The topology of MANET is represented by $G(V, E)$, where $V$ is the set of nodes and $E$ is the set of edges. Each node $u \epsilon V$ can communicate directly with nodes lying in its transmission range. A set of these one-hop neighbours of node $u$ are represented by $N^{1}(u) \subseteq V$. In OLSR, each node $u \epsilon V$ selects a subset of its one-hop neighbour nodes, known as MPRs of $u$, such that $M P R(u) \subseteq N^{1}(u)$ for relaying its traffic to the distant nodes. OLSRv2 provides two kinds of MPR nodes, i.e. Routing MPRs and Flooding MPRs. The former are used to 
forward data packets of the selector nodes and latter is used to relay topology control information to other nodes in the network.

Each node $u \in V$, has two kinds of willingness value associated with it, Flooding Willingness $W_{F}(u)$ and Routing Willingness $W_{R}(u)$. $W_{F}(u)$ represents how willing a node is to be selected as a Flooding MPR. Similarly, $W_{R}(u)$ gives willingness of a node to be selected as a Routing MPR. Each node $u$ propagates its $W_{F}(u)$ and $W_{R}(u)$, both of which vary from 0 (WILL_NEVER) to 15 (WILL_ALWAYS), to its one-hop neighbours through control messages (HELLO messages). In OLSRv2, a node selects its MPRs based upon willingness, reachability and degree of its one-hop neighbour nodes. A major limitation of OLSRv2 is that it does not provide any mechanism for computing the willingness value (both $W_{F}(u)$ and $W_{R}(u)$ ) for a node. The willingness value for each node is set to WILL_DEFAULT, i.e. 7. ES-MPR provides a new mechanism for computation of both $W_{F}(u)$ and $W_{R}(u)$ by taking into account both energy and security metrics.

\subsection{Selection Process}

ES-MPR introduces the novel concept of Composite Eligibility Index (CEI) which examines the eligibility of a node for being selected as MPR. CEI is determined by the MPR selector node based on energy and security parameters, whereas willingness is computed and propagated by the node itself. CEI is used in conjunction with received willingness of a node to determine 'Selection Parameter' which is a fundamental factor for MPR selection in ES-MPR.

\subsubsection{Willingness}

In ES-MPR, each node computes its willingness value (both $W_{F}(u)$ and $W_{R}(u)$ ) on the basis of metrics: Lifetime Metric $M_{L T}$, Available Bandwidth Metric $M_{A B}$, Queue Occupancy Metric $M_{Q O}$. Different weights are assigned to these metrics to determine the value of Flooding Willingness and Routing Willingness.

\section{- Lifetime Metric $M_{L T}$}

A node's lifetime is the duration for which it is operative before its residual energy drains out. Lifetime of individual nodes has direct impact on network operational lifetime. The lifetime of a node $u$ depends upon its residual energy and along with its drain rate [23] at time' $t$ ' and is given as:

$$
L T(t)=\frac{R E(t)}{D R(t)}
$$

The lifetime metric used for willingness computation is given by:

$$
M_{L T}=\frac{L T(t)}{L T_{\max }}
$$

where $L T_{\max }$ is the maximum value of a node's lifetime.

\section{- Available Bandwidth Metric $M_{A B}$}

ES-MPR takes into account the available bandwidth of nodes for MPR selection. A node's available bandwidth can be computed as: 


$$
A B=L B^{*} I T
$$

where $L B$ is the link bandwidth and $I T$ is the time for which the node is in idle state. Available Bandwidth Metric used for willingness computation is given as:

$$
M_{A B}=\frac{A B}{A B_{\max }}
$$

where $A B_{\max }$ is the maximum value for a node's available bandwidth.

\section{- Queue Occupancy Metric $M_{Q O}$}

A node buffers the received packets in its queue before forwarding them to the data link layer. Once the queue is full, upcoming packets are dropped by the node. This leads to energy loss of the sending nodes whose packets are dropped due to fully occupied queue. Moreover, average queue occupancy is directly proportional to the end-to-end delay in the network. Therefore, ES-MPR uses the complement of average queue occupancy at a node as a metric for willingness computation. The greater the value of average queue occupancy at a node, the lower will be its willingness value. It is computed at a regular interval of 2 seconds.

Queue Occupancy metric for determining willingness is given as:

$$
M_{Q O}=1-\frac{Q O_{\text {avg }}}{Q O_{\max }}
$$

where $Q O_{\max }$ is the maximum capacity of queue at a node.

ES-MPR assigns different weights to all three metrics, i.e. $M_{L T}, M_{A B}$ and $M_{Q O}$ for computation of Flooding Willingness $W_{F}(u)$ and Routing Willingness $W_{R}(u)$ of a node $u$.

$$
\begin{gathered}
X_{F}(u)=\alpha * M_{L T}+\beta * M_{A B}+\gamma * M_{Q O} \\
W_{F}(u)=X_{F}(u) * W_{\max }
\end{gathered}
$$

$X_{F}(u)$ is the weighted additive function of all the above mentioned metrics having weights $(\alpha=0.4, \beta=0.2, \gamma=0.4)$. $W_{\max }$ is the maximum willingness value of a node, i.e. 15 .

$$
\begin{aligned}
X_{R}(u)= & \alpha^{\prime} * M_{L T}+\beta^{\prime *} M_{A B}+\gamma^{\prime} * M_{Q O} \\
& W_{R}(u)=X_{R}(u) * W_{\max }
\end{aligned}
$$

$X_{R}(u)$ is the weighted additive function of the three metrics having weights $\left(\alpha^{\prime}=0.5, \beta^{\prime}=0.3\right.$, $\left.\gamma^{\prime}=0.2\right)$. The considered metrics are assigned different weights for computation of $W_{F}(u)$ and $W_{R}(u)$. The weights assigned to the metrics depend upon different requirements of flooding and routing MPRs. For determining $W_{R}(u)$ of a node $u, M_{L T}$ and $M_{A B}$ are given more weight as compared to $M_{Q O}$. As data packets are larger in size relative to control messages, routing of data packets consumes more energy and bandwidth compared to spreading control information in the network.

On the other hand, for determining $W_{F}(u)$ of a node $u$, more weight is assigned to $M_{Q O}$ as compared to $M_{A B}$ to ensure speedy delivery of topology control information to other nodes in the network. As topology in MANETs is dynamic and changes rapidly due to mobility of nodes, it is imperative to avoid any kind of delay in propagation of topology control information. Such delays may lead to inconsistency between the node's view of network 
topology and actual network scenario. Furthermore, weights assigned to these metrics are flexible and can be tuned as per network requirements.

\subsubsection{Composite Eligibility index (CEI)}

In ES-MPR, each node $u$ computes the Composite Eligibility Index for every node $v \in N^{1}(u)$ based upon the factors: Misbehaving Probability $P_{M}(v)$, Forwarding Behaviour $F B(v)$, Power Factor $P F(v)$, Hello Loss Ratio $H^{L}(v)$ and Stability Index $S I(v)$. CEI is determined by the MPR selector for all its one-hop neighbours. It signifies how eligible a node is to be selected as an MPR. ES-MPR provides two kinds of Composite Eligibility Index, i.e. $C E I_{F}(v)$ and $C E I_{R}(v)$, each of which determines the suitability of a node for being selected as Flooding MPR and Routing MPR respectively. The value of CEI varies from 0 to 15 $\left(C E I_{\max }\right)$. The greater the value of CEI of a node, the higher is the chance of it being selected as an MPR.

\section{- Misbehaving Probability}

Due to limitation of energy resources available with the nodes, in MANETs, they tend to misbehave by dropping packets of other nodes which are meant to be forwarded, with an aim of conserving its valuable resources, like energy. Nodes, in ES-MPR, employ an overhearing mechanism to check whether the packets are successfully forwarded by the neighbour node or not. After transmitting a packet to node $v$, node $u$ overhears the wireless channel for a fixed duration of time $T_{o}(500 \mathrm{~ms})$ to check if node $v$ forwards the packet or not. If node $u$ hears an attempt to transfer the packet by node $v$ within time $T_{o}$, then the packet is considered to be forwarded. If node $v$ does not forward the packet within time $T_{o}$, it is considered as dropped.

Node $u$ determines misbehaving probability of node $v, P_{M}(v)$ as follows:

$$
P_{M}(v)=\frac{\text { No. of packets of } \mathrm{u} \text { dropped by } \mathrm{v}}{\text { Total packets sent by u to } \mathrm{v}}
$$

$P_{M}(v)$ of a node $v$ is dynamically updated depending upon its communication with the node $u$. The complement of misbehaving probability is used to determine the value of CEI:

$$
=1-P_{M}(v)
$$

\section{- Forwarding Behaviour}

Another parameter used for computation of CEI is based on the forwarding behaviour of nodes. Forwarding behaviour of a node $v$ as determined by node $u$ is given by:

$$
F B(v)=\frac{P(v, u)}{P(v, u)+P(u, v)}
$$

where $P(v, u)$ is the number of packets forwarded by node $v$ on the behalf of node $u$. Similarly, $P(u, v)$ is the number of packets node $u$ has forwarded for node $v$.

This metric gives the fraction of packets forwarded by node $v$ of node $u$ to the total number of packets transferred between node $u$ and node $v$. The greater the value of $F B$, more eligible the node is for selection as MPR. 


\section{- Power Factor}

ES-MPR takes into account Power Factor for MPR selection based upon the energy of the power amplifier required by the MPR selector to transmit packets to MPR nodes. Nodes can adjust their transmission power and minimum power required by node $u$ to communicate with node $v$ is determined by algorithm proposed in [24] [25]. Power Factor used for computation of CEI is given as:

$$
P F(v)=1-\frac{T P(v)_{a v g}}{T P_{\max }}
$$

where $T P(v)$ is the transmission power required to communicate with node $v$ and $T P_{\max }$ is the maximum allowable transmission power. Taking Power Factor into consideration ensures energy efficient MPR selection. Nodes that require less transmission power of MPR selector node are preferred to the nodes that require higher transmission power for communication. Selecting MPR nodes that require low transmission power reduces the energy consumption of MPR selector nodes, in turn, increasing the operational lifetime of the network.

\section{- Hello Loss Ratio}

For energy efficient MPR selection, it is imperative to examine stability of connection between MPR selector and MPR nodes. Mobile nodes, in MANETs, are continuously manoeuvring in and out of each other's transmission range. Mobility of nodes during communication may lead to packet loss which, in turn, results in energy loss at the sender node. In OLSR, nodes exchange neighbour sensing messages in the form of HELLO messages. Each node $u \epsilon V$ broadcasts HELLO message which is received by its one-hop neighbour $v \in N^{1}(u)$ at a regular interval, known as HELLO Interval $H_{\text {interval. }}$ HELLO messages could be received by only those nodes that lie within the transmission range of the sender.

ES-MPR exploits this concept to examine the relative stability between nodes in the form of Hello loss ratio. This is done by recording the number of HELLO messages received and the total number of expected HELLO messages to determine the frequency of HELLO messages lost. Hello loss ratio determined by node $u$, of node $v$, is given by:

$$
\begin{gathered}
H^{L}(v)=\frac{H_{\text {exp }}(v)-H_{\text {recv }}(v)}{H_{\text {exp }}(v)} \\
H_{\text {exp }}(v)=\frac{\text { current time - first HELLO time }}{H_{\text {interval }}}
\end{gathered}
$$

$H_{\text {exp }}(v)$ is the number of HELLO messages that are expected to be received from node $v$. The complement of hello loss ratio is used for computation of CEI:

$$
=1-H^{L}(v)
$$

\section{- Stability Index}

In order to determine stability of link between MPR selector and MPR node, Bienayme-Chebyshev inequality theory [10] is applied to compute the variance in signal 
strengths received the neighbour node. The computed variance value shows how stable is the link between MPR selector and neighbour node. Lesser the variance more is the relative stability between the corresponding nodes. Variance in signal strength $S V(u, v)$ of node $v$ as computed by node $u$ is given by:

$$
S V(u, v)=\left(\frac{\sum_{i} S S_{v i}^{2}}{n}\right)-\left(\frac{\sum_{i} S S_{v i}}{n}\right)^{2}
$$

where $S S_{v}$ is the signal strength of node $v$ received by $u$ and $n$ is the total number of messages received by $u$ of node $v$. In ES-MPR, a node assigns Link Stability Score to all its one-hop neighbours based upon the computed signal variance of nodes. The link stability score assigned to the node is inversely proportional to its signal variance value. In other words, more the signal variance, less stable will be the link and hence, lower the link stability score of the node. The value of link stability score ranges from 1 to $N_{O H}$, where $N_{O H}$ is the total number of one-hop neighbours of the MPR selector node.

Stability Index used for computation of CEI is determined as:

$$
S I(v)=\frac{L S S_{v}}{N_{O H}}
$$

Fig. 2 depicts Signal Variance and Link Stability Score computed by Node $S$ for all its one-hop neighbours. As the number of neighbours of $S$ is equal to 6, i.e. $N_{O H}=6$, therefore link stability score, in this case, ranges from 1 to 6 . As signal variance of link $S V(S, D)$ is 0.3 , i.e. minimum out of all the neighbours, therefore $\operatorname{LSS}_{D}(6)$ is maximum for Node $D$. On the other hand, signal variance $S V(S, A)(1.7)$ is maximum, hence node $A$ has minimum link stability score, i.e. $L S S_{A}=1$.

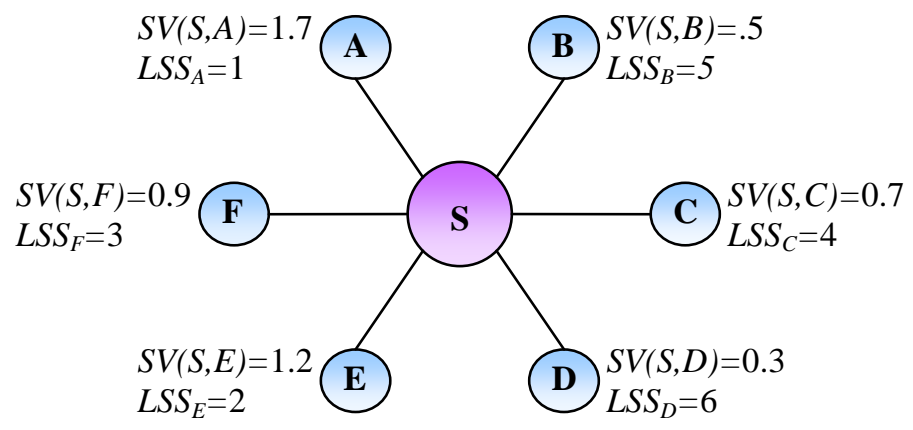

Fig. 2. Signal variance and link stability score

On the basis of above discussed parameters both $C E I_{F}(v)$ and $C E I_{R}(v)$ are determined for selection of Flooding and Routing MPRs respectively.

$$
\begin{gathered}
Y_{F}(v)=\omega_{1}^{*}\left(1-P_{M}(v)\right)+\omega_{2} * F B(v)+\omega_{3} * P F(v)+\omega_{4} *\left(1-H^{L}(v)\right)+\omega_{5} * S I(v) \\
C E I_{F}(v)=Y_{F}(v) * C E I_{\text {max }}
\end{gathered}
$$


where $\omega_{1}=0.2 \omega_{2}=0.1 \omega_{3}=0.2 \omega_{4}=0.2 \omega_{5}=0.3$ are the weights assigned to different parameters. $C E I_{\max }$ is the maximum value of Composite Eligibility Index, i.e. 15.

$$
\begin{gathered}
Y_{R}(v)=\omega_{1}^{\prime} *\left(1-P_{M}(v)\right)+\omega_{2}^{\prime} * F B(v)+\omega_{3}^{\prime} * P F(v)+\omega_{4}^{\prime} *\left(1-H^{L}(v)\right)+\omega_{5}^{\prime} * S I(v) \\
C E I_{R}(v)=Y_{R}(v) * C E I_{\text {max }}
\end{gathered}
$$

where $\omega_{1}^{\prime}=0.3 \quad \omega_{2}^{\prime}=0.1 \omega_{3}^{\prime}=0.3 \omega_{4}^{\prime}=0.1 \omega_{5}^{\prime}=0.2$ are the weights assigned to above mentioned parameters.

For computing $C E I_{R}(v)$, more weights are assigned to metric based on Misbehaving Probability and Power Factor than in computation of $C E I_{F}(v)$. As the amount of data traffic is more than control traffic, misbehaving node tends to drop more data packets than control packets in order to conserve its energy. The energy consumed in data packet transmission is higher relative to energy required for transmission of control packets; therefore, selecting an MPR which requires low transmission power helps to optimize energy consumption in the network. On the other hand, for computing $C E I_{F}(v)$ more weights are assigned to Stability Index and Hello Loss Ratio to avoid delay in transmission of control information which may lead to inconsistent topology information at nodes. Furthermore, the weights assigned can be attuned according to system requirements.

\subsubsection{Selection Parameter}

Each node $u \in V$ uses willingness along with CEI to compute the value of Selection Parameter SP for its neighbour nodes $\forall v \in N^{1}(u)$. Unlike existing schemes which depend upon willingness as fundamental parameter for MPR selection, ES-MPR performs energy efficient secure MPR selection based upon node's Selection Parameter, degree and reachability. Similar to willingness and CEI in ES-MPR, separate Selection Parameter $S P_{\mathrm{F}}(v)$ and $S P_{\mathrm{R}}(v)$ are determined for Flooding and Routing MPR respectively.

$$
\begin{aligned}
& S P_{F}(v)=\phi^{*} C E I_{F}(v)+(1-\phi) * W_{F}(v) \\
& S P_{R}(v)=\phi^{*} C E I_{R}(v)+(1-\phi) * W_{R}(v)
\end{aligned}
$$

where $\phi=0.6$ and (1- $\phi)$ are the weights given to Composite Eligibility Index and willingness respectively. More weight is assigned to CEI as it is calculated by the selector node, whereas willingness is computed and broadcasted by neighbour nodes themselves.

Fig. 3 shows the actions performed by MPR selector node and neighbour nodes in ES-MPR. Reachability $R$ of a node gives the number of two-hop neighbours which are reachable only through that node. Degree $D$ is the number of symmetric one hop neighbours of the respective node. The details about Reachability $R$ and Degree $D$ of a node are given in [3]. Algorithm 1 presents the steps involved in selection of Flooding and Routing MPRs. A node with high value of $\operatorname{SP}(v)$ (i.e. $S P_{\mathrm{F}}(v)$ or $\left.S P_{\mathrm{R}}(v)\right)$ is a desirable candidate for MPR selection. Therefore, nodes having $\mathrm{SP}(v)>=14$ are given highest priority and are directly added to MPR set. 

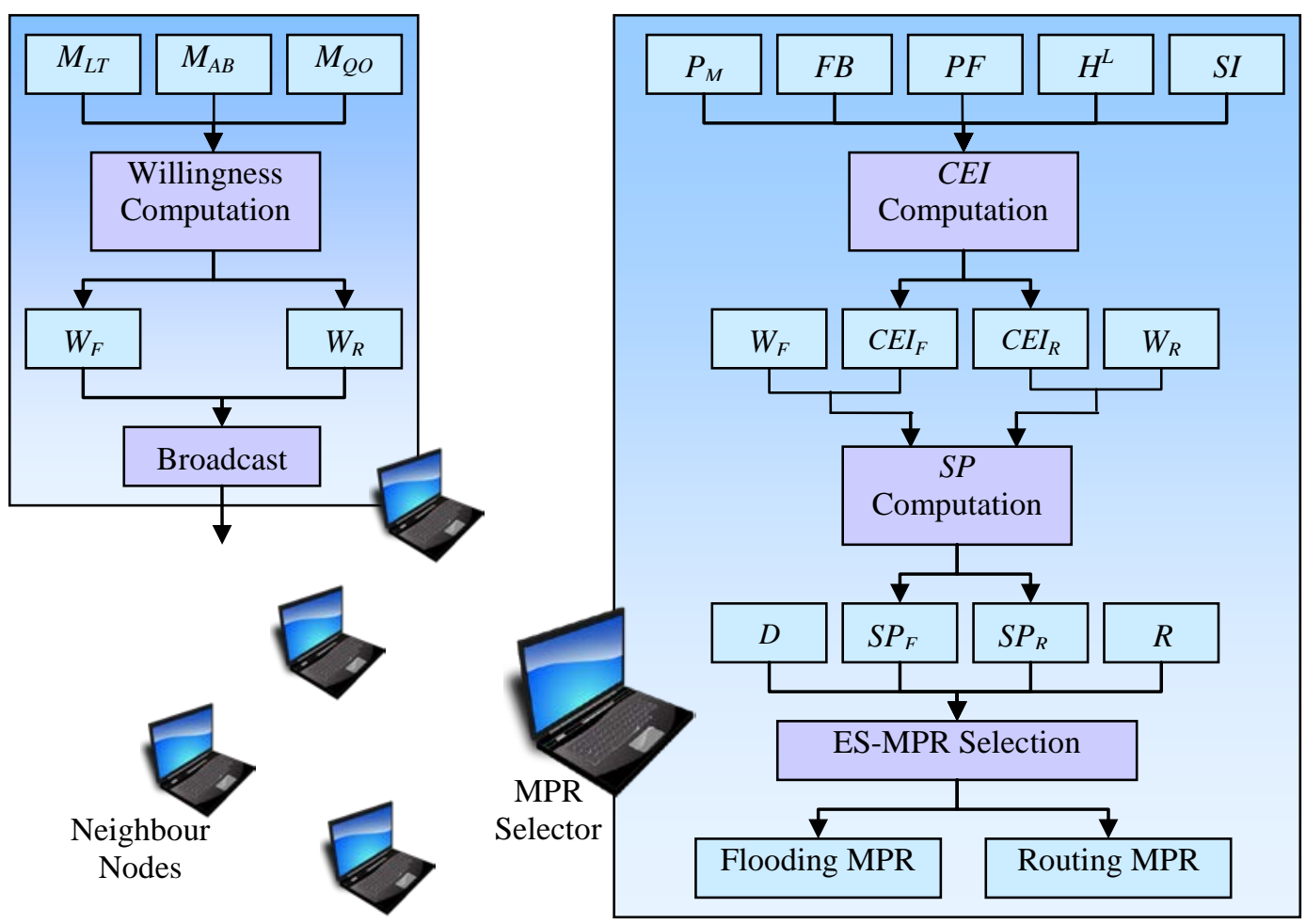

Fig. 3. Energy efficient secure MPR selection.

\subsection{Incentive Mechanism for MPR nodes}

In ES-MPR, MPR selector $u$ employs Incentive Mechanism to provide incentives to each of its selected MPR nodes, $v \in M P R(u)$. The incentive is provided in the form of 'chips' according to the forwarding behaviour of the MPR nodes. Every node $u \epsilon V$ keeps a record of the number of packets forwarded, $P(v, u)$ by each of its one-hop neighbour $v \in N^{1}(u)$ on behalf of node $u$. Similarly, it accounts for the number of packets $P(u, v)$ that are forwarded by node $u$ on behalf of node $v$. Each node $u \epsilon V$ maintains a 'chip counter' for each of its neighbour node $v \in N^{1}(u)$. The value of chip counter for node $v$ is incremented when node $u$ overhears a successful forwarding attempt by node $v$. Whereas chip counter value for node $v$ is decremented when node $u$ relays a packet on behalf of node $v$. Node $u$ forwards the packets of node $v$, until its chip counter reaches zero. Once the chip counter value of node $v$, at node $u$, reaches zero all the forwarding requests by $v$ are rejected by node $u$.

ES-MPR employs this mechanism for handling of misbehaving nodes by enforcing them to participate in the network and concede their misbehaviour. As in order to transmit its data in the network, a node has to maintain a non-zero chip counter by forwarding packets on behalf of other nodes. The value of chip counter, for every node, is initialized to default value (200) in order to begin communication. Furthermore, chip counter for every node is 
incremented by a small amount $C_{o}$ (set to 5), at regular interval $T_{C}(10 \mathrm{sec}$ ), to avoid deadlock situation in which nodes stop forwarding each others' packets due to lack of chips.

ES-MPR provides special incentives $\left(I_{M P R}\right)$ to the selected MPR nodes to encourage nodes for MPR selection. The chips provided to the MPR nodes at regular interval $T_{C}$, are scaled according to the forwarding behaviour of MPR nodes.

$$
I_{M P R}=\left(1+\frac{P(v, u)}{P(u, v)+P(v, u)}\right) * C_{o}
$$

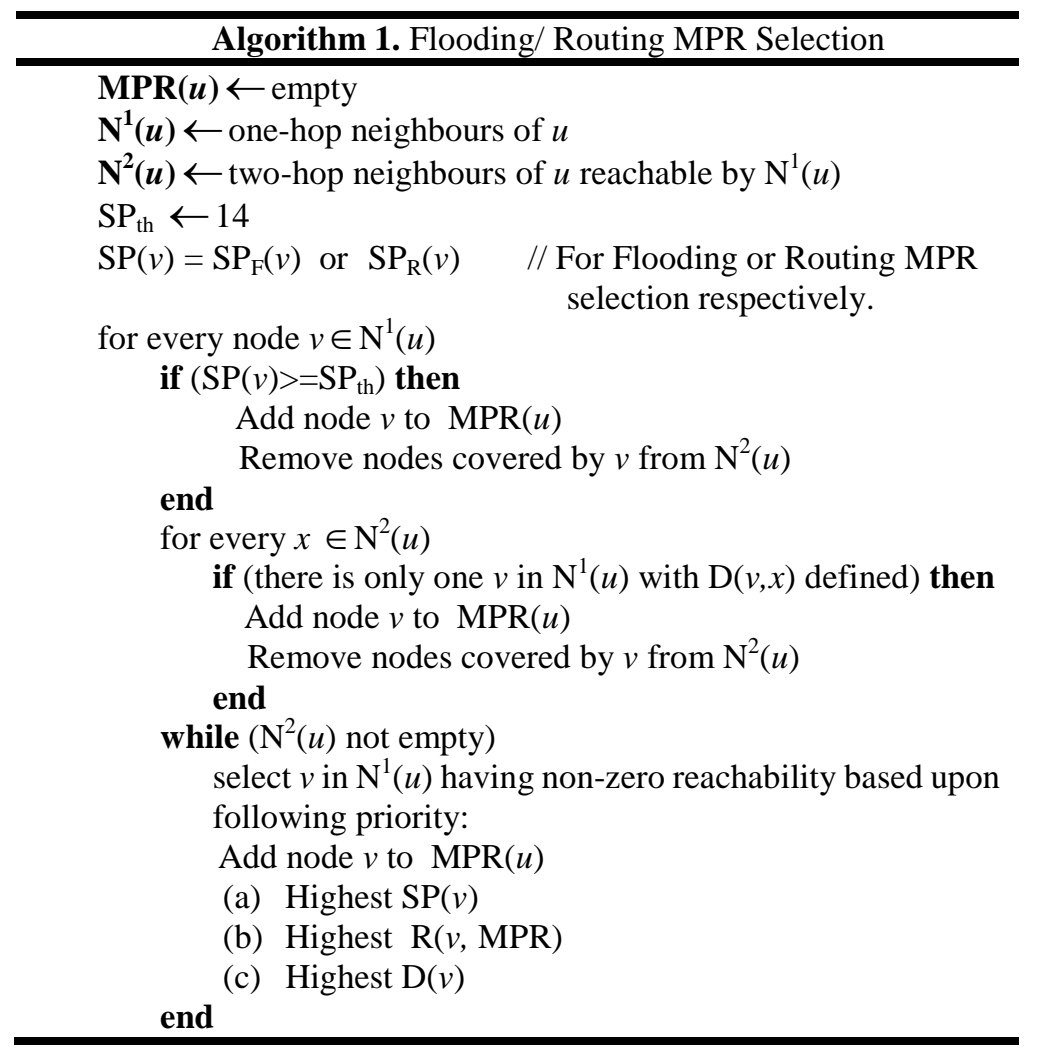

\section{Simulation and Performance Evaluation}

The network simulator NS-2 [26] is used to simulate the network for performance evaluation. Mobility of nodes is based on Random Way-Point Mobility Model. The simulation procedure has been repeated 25 times to achieve 95 percent confidence level and the average values are plotted for each data point. The energy model given in [25] is used to determine energy consumption in the network. Table 3 lists the various simulation parameters. 
Table 3. Fixed and constant parameters for simulation

\begin{tabular}{|l|l|l|l|}
\hline \multicolumn{1}{|c|}{ Parameters } & \multicolumn{1}{c|}{ Value } & \multicolumn{1}{c|}{ Parameters } & \multicolumn{1}{c|}{ Value } \\
\hline Area & $500 \mathrm{X} 500 \mathrm{~m}^{2}$ & Initial Energy & $100 \mathrm{~J}$ \\
\hline Network Nodes & 50 & Transmission Power & 10 to $100 \mathrm{~mW}$ \\
\hline Transmission Range & $100 \mathrm{~m}$ & Sending Energy & 0.042 to $0.084 \mathrm{~J}$ \\
\hline Speed & 0 to $30 \mathrm{~m} / \mathrm{s}$ & Receiving Energy & $0.04 \mathrm{~J}$ \\
\hline Data Rate & $100 \mathrm{Kbps}$ & Idle Energy & $0.01 \mathrm{~J}$ \\
\hline Packet Size & 512 Bytes & Overhearing Energy & $0.04 \mathrm{~J}$ \\
\hline
\end{tabular}

\subsection{Simulation Results}

Performance of ES-MPR is evaluated by comparing it with other MPR selection techniques namely ELSQ [11], EE-OLSR [5] and REOLSR2 [6]. The results obtained from simulation are as follows:

Fig. 4 illustrates the effect of increasing percentage of misbehaving nodes on Packet Delivery Ratio (PDR) of ES-MPR and other deployed algorithms. It can be observed that ES-MPR outperforms other schemes, in terms of PDR, in the presence of misbehaving nodes in the network. This is due to its ability to handle and avoid misbehaving nodes during MPR selection. ELSQ gives the second best PDR as it takes into account link stability along with queue occupancy which reduces packet loss. EE-OLSR and REOLSR2 give low PDR values as they only consider lifetime and residual energy respectively, and therefore fail to perform in hostile environment.

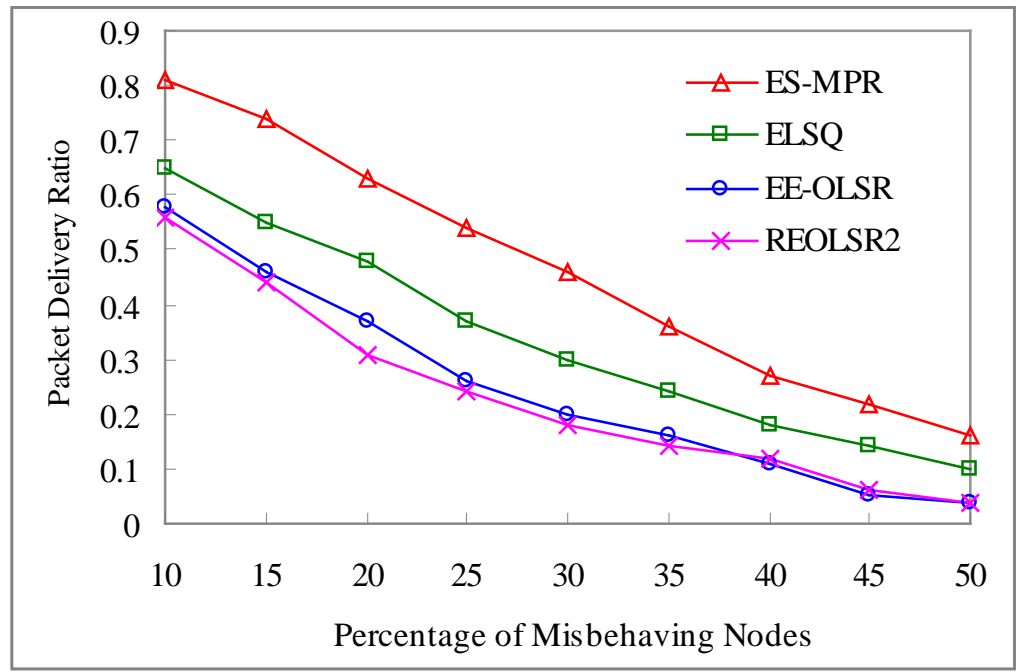

Fig. 4. Packet delivery ratio of ES-MPR and other schemes. 
Fig. 5 highlights the network lifetime for ES-MPR and various other schemes with respect to the percentage of misbehaving nodes in the network. Network Lifetime is defined as the time when the first dead node is detected in the network.

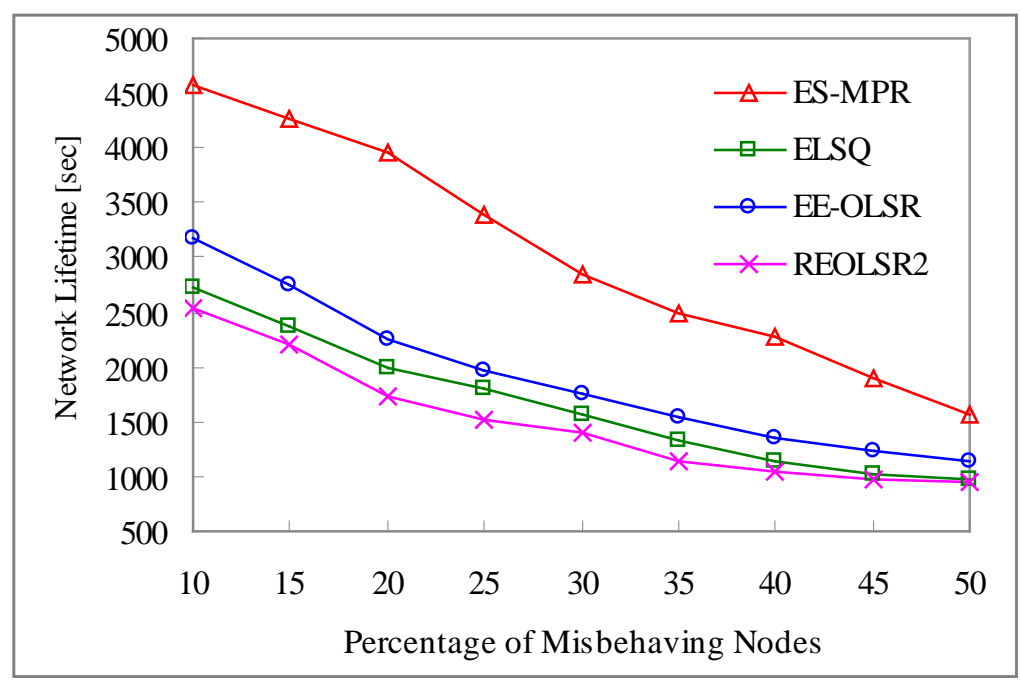

Fig. 5. Network lifetime of ES-MPR and other considered schemes.

As seen in Fig. 5, ES-MPR shows highest network lifetime as compared to the other considered algorithms. This is due to the consideration given to transmission power, lifetime, stability and security measure during MPR selection. Next best performing algorithm is EE-OLSR as it takes into account drain rate of the nodes along with their residual energy. ELSQ and REOLSR2 exhibit low network lifetime in the presence of misbehaving nodes.

Fig. 6 displays the average energy consumption per packet with increasing percentage of misbehaving nodes in the network for ES-MPR and other algorithms. ES-MPR provides minimum energy consumption per packet as compared to all other considered algorithms owing to consideration of transmission power required to communicate with MPR node. ES-MPR selects MPRs which require low transmission power, in turn, decreasing the average energy consumption per packet. 


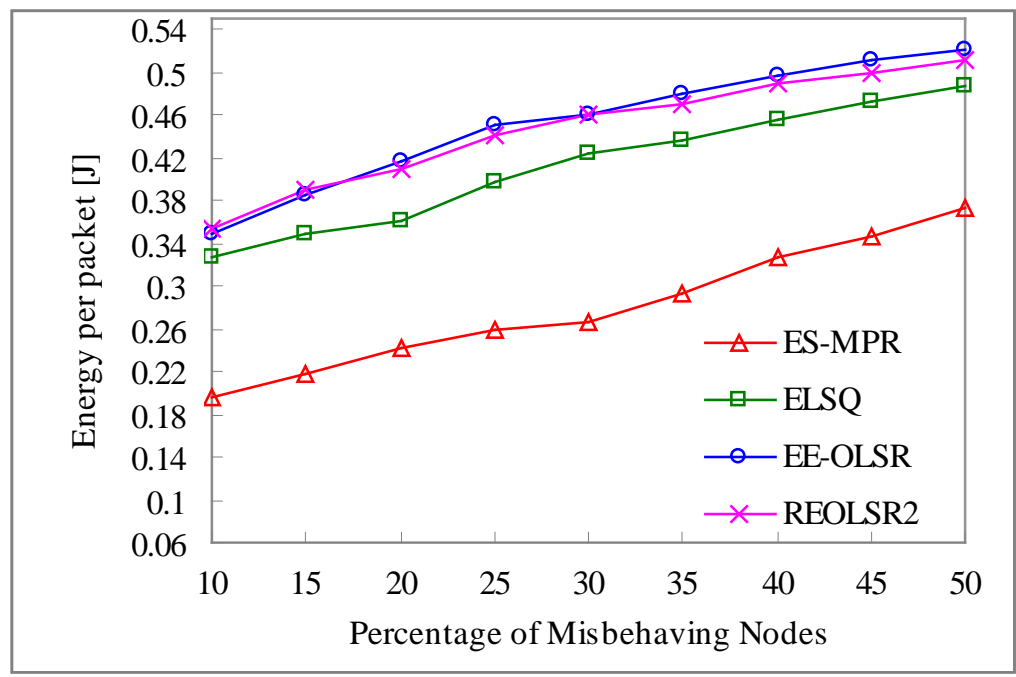

Fig. 6. Energy consumed per packet in ES-MPR and other schemes

Fig. 7 illustrates the efficiency of ES-MPR in choosing MPRs, thereby decreasing the total number of different MPR nodes selected for varying number of nodes in the network. ES-MPR selects the minimum number of MPRs as compared to other deployed schemes. Energy and security metrics employed in ES-MPR ensure the selection of stable MPRs, hence forming a secure, reliable and durable topology in the network.

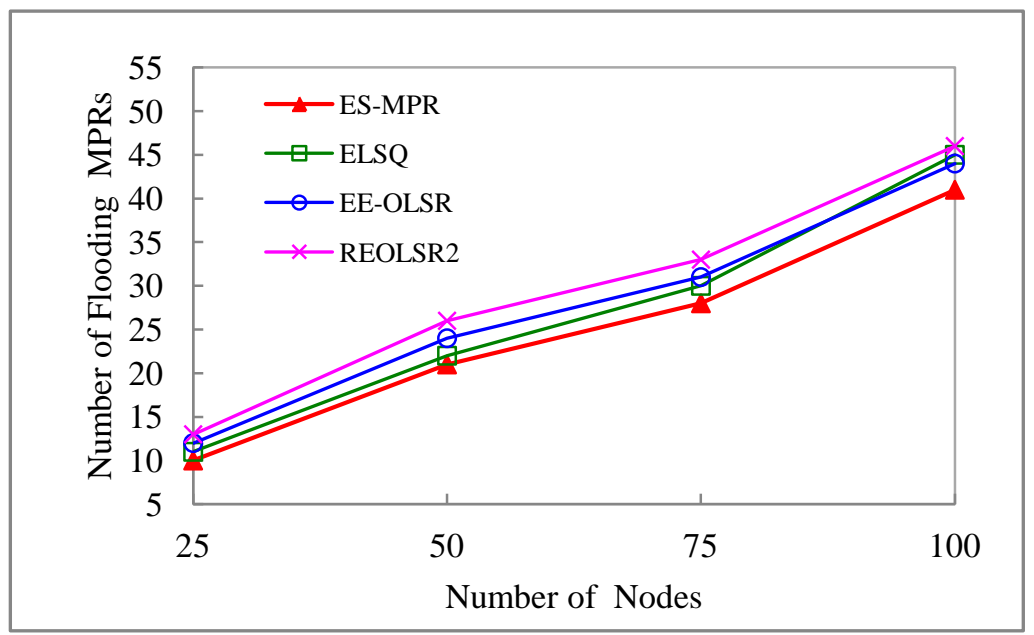

Fig. 7. Number of Flooding MPRs in ES-MPR and other schemes 


\section{Conclusion}

In OLSR, Multipoint Relays (MPRs) are selected by nodes to relay their data/control traffic through the network. Existing MPR selection schemes are based on energy and QoS metrics such as residual energy, link stability, lifetime etc. However, these schemes do not take into account any security metrics, hence may fail to perform in hostile environment. This paper proposes an energy efficient secure MPR selection (ES-MPR) mechanism which considers various energy metrics and misbehaviour of nodes for MPR selection process. It computes 'Composite Eligibility Index' of nodes (based upon misbehaving probability, Power Factor, Stability Index, Hello Loss Ratio and Forwarding Behaviour) which is combined with the willingness value (calculated using Lifetime, Available Bandwidth and Queue Occupancy) to provide separate selection parameters for Flooding and Routing MPRs. The performance of ES-MPR is evaluated using NS-2 by comparing it with existing MPR selection techniques. ES-MPR outperforms existing schemes in terms of network lifetime, PDR and other metrics due to its ability to provide energy efficient secure and stable MPRs, thereby forming durable and reliable topology in the network.

\section{References}

[1] D.B. Johnson, D.A. Maltz and J. Broch, "DSR: The Dynamic Source Routing protocol for multi-hop wireless ad hoc networks," Ad Hoc Networking, Chapter 5, edited by C.E. Perkins, Addison-Wesley, pp. 139-172, 2001.

[2] R. Malekian, A. Karadimce and A. H. Abdullah, "AODV and OLSR routing protocols in MANET," in Proc. of IEEE Thirty-third International Conference on Distributed Computing Systems Workshops, pp. 286-289, 8-11 July 2013. Article (CrossRef Link).

[3] T. Clausen and P. Jacquet, “Optimized Link State Routing Protocol (OLSR),” RFC Editor, 2003.

[4] T. Clausen, C. Dearlove, P. Jacquet and U. Herberg, "Optimized Link State Routing Protocol version 2,” Internet Engineering Task Force (IETF) RFC 7181, April 2014.

[5] F. De Rango, M. Fotino and S. Marano, "EE-OLSR: Energy Efficient OLSR routing protocol for Mobile ad-hoc Networks," in Proc. of IEEE Military Communications Conference, MILCOM 2008, pp. 1-7, 16-19 November 2008. Article (CrossRef Link).

[6] W.K. Hirata, Y. Higami and S.Y. Kobayashi, "Residual energy-based OLSR in mobile ad hoc networks," in Proc. of International Conference on Multimedia Technology (ICMT), pp. 3214-3217, 26-28 July 2011. Article (CrossRef Link).

[7] J. M. Robert, H. Otrok and A. Chriqi, "RBC-OLSR: Reputation-based Clustering OLSR Protocol for Wireless Ad Hoc Networks,” Computer Communications, vol. 35, no. 4, pp. 487-499, February 2012. Article (CrossRef Link).

[8] N. Dashbyamba, C. Wu, S. Ohzahata and T. Kato , "An improvement of OLSR using fuzzy logic based MPR selection," in Proc. of Fifteenth Asia-Pacific Network Operations and Management Symposium (APNOMS), pp.1-6, 25-27 September 2013.

[9] A. Kots and M. Kumar, "The fuzzy based QMPR selection for OLSR routing protocol," Wireless Networks. vol. 20, no. 1, pp. 1-10, January 2014. Article (CrossRef Link). 
[10] A. Moussaoui, F. Semchedine, A. Boukerrama, "A link-state QoS routing protocol based on link stability for Mobile Ad hoc Networks," Journal of Network and Computer Applications, vol. 39, pp. 117-125, March 2014. Article (CrossRef Link).

[11] R. B. Patil and A.B. Patil, "Energy, Link Stability and Queue Aware OLSR for Mobile Ad Hoc Networks," in Proc. of International Conference on Advances in Computing, Communications and Informatics, pp. 1020-1025, 10-13 August 2015. Article (CrossRef Link).

[12] W. A. Jabbar, M. Ismail and R. Nordin, "Multi-criteria based multipath OLSR for battery and queue-aware routing in multi-hop ad hoc wireless networks," Wireless Networks, vol. 21, no. 4, pp. 1309-1326, May 2015. Article (CrossRef Link).

[13] L. Zheng, Y. Nenghai and D. Zili, "NFA: A New Algorithm to Select MPRs in OLSR,” in Proc. of Fourth International Conference on Wireless Communications, Networking and Mobile Computing, WiCOM '08, pp.1-6, 12-14 October 2008. Article (CrossRef Link).

[14] H. Badis, Hakim and K. Al Agha, "QOLSR, QoS routing for ad hoc wireless networks using OLSR,” European Transactions on Telecommunications, vol. 16, no. 5, pp. 427-442, October 2005. Article (CrossRef Link).

[15] T. Koga, S. Tagashira, T. Kitasuka, T. Nakanishi and A. Fukuda, "Highly efficient multipoint relay selections in link state QoS routing protocol for multi-hop wireless networks," in Proc. of IEEE International Symposium on World of Wireless, Mobile and Multimedia Networks \& Workshops, WoWMoM 2009 , pp.1-9, 15-19 June 2009. Article (CrossRef Link).

[16] H. Chizari, M. Hosseini, S. Salleh, S.A. Razak and A.H. Abdullah, "EF-MPR, a new energy efficient multi-point relay selection algorithm for MANET," The Journal of Supercomputing, vol. 59, no. 2, pp-744-761, 2012. Article (CrossRef Link).

[17] T.H. Lin, H.C. Chao and I. Woungang, "An Enhanced MPR-Based Solution for Flooding of Broadcast Messages in OLSR Wireless ad hoc Networks," Mobile Information Systems, vol. 6, no. 3, pp. 249-257, 2010. Article (CrossRef Link).

[18] R.D. Joshi and P.P. Rege, "Implementation and analytical modelling of modified optimised link state routing protocol for network lifetime improvement," IET Communications, vol.6, no.10, pp.1270-1277, July 3 2012. Article (CrossRef Link).

[19] A. Boushaba, A. Benabbou, R. Benabbou, A. Zahi and M. Oumsis, "Multi-point relay selection strategies to reduce topology control traffic for OLSR protocol in MANETs," Journal of Network and Computer Applications, vol. 53, pp. 91-102, July 2015. Article (CrossRef Link).

[20] A. Chriqi, H. Otrok and J.-M. Robert, "SC-OLSR: Secure Clustering-Based OLSR Model for Ad Hoc Networks," in Proc. of IEEE International Conference on Wireless and Mobile Computing, Networking and Communications, WIMOB 2009, pp.239-245, 12-14 October 2009. Article (CrossRef Link).

[21] A. Mehmood and H. Song, "Smart Energy Efficient Hierarchical Data Gathering Protocols for Wireless Sensor Networks,” Smart Computing Review, vol. 5, no. 5, pp.425-462, October 2015. Article (CrossRef Link).

[22] D. S. J. De Couto, D. Aguayo, J. Bicket and R. Morris, "A High-throughput Path Metric for Multi-hop Wireless Routing,” Wireless Networks, vol. 11, no. 4, pp-419-434, July 2005. Article (CrossRef Link).

[23] K. Dongkyun, J.J. Garcia-Luna-Aceves, K. Obraczka, J.C. Cano, and P. Manzoni, "Routing mechanisms for mobile ad hoc networks based on the energy drain rate," IEEE Transactions on Mobile Computing, vol. 2, no. 2, pp. 161-173, April 2003. Article (CrossRef Link).

[24] A. Sheth and R. Han , "Adaptive power control and selective radio activation for low-power infrastructure-mode 802.11 LANS," in Proc. of the Twenty Third International Conference on Distributed Computing Systems, pp. 812-818, 19-22 May 2003. Article (CrossRef Link). 
[25] Q. Wang, M. Hempstead and W. Yang, "A Realistic Power Consumption Model for Wireless Sensor Network Devices," in Proc. of Third Annual IEEE International Conference on Sensor and Ad Hoc Communications and Networks, SECON 2006, pp. 286-295, 28 September 2006. Article (CrossRef Link)

[26] T. Issariyakul and E. Hossain, "Introduction to network simulator NS2," $2^{\text {nd }}$ Ed. USA, Springer, 2009. Article (CrossRef Link).

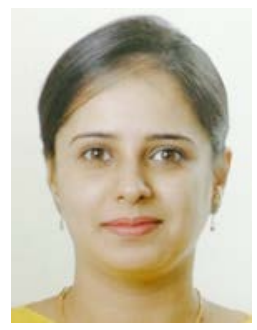

Anjali Anand is pursuing Ph.D. from Department of Computer Engineering, Punjabi University, Patiala. She has done M.Tech. from Punjabi University, Patiala. She has contributed 6 articles in various research journals. Her areas of interest are Computer Networks, Mobile Ad hoc Networks. Anjali Anand can be contacted at: anjalianand_87@yahoo.in

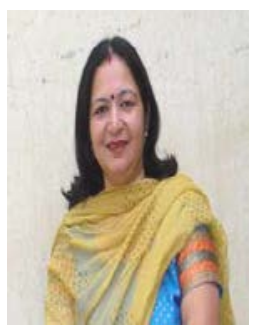

Dr. Rinkle Rani is working as Assistant Professor in Computer Science and Engineering Department, Thapar University, Patiala since 2000. She has done her Post graduation from BITS, Pilani and Ph.D. from Punjabi University, Patiala in the area of Computer Networks. She has more than 18 years of teaching experience. She has supervised 34 M.Tech. Dissertations and contributed 50 articles in Conferences and 41 papers in Research Journals. Her areas of interest are Computer Networks and Big data mining and Analysis. She is member of professional bodies: ACM, IEEE, ISTE and CSI. She may be contacted at: raggarwal@ thapar.edu

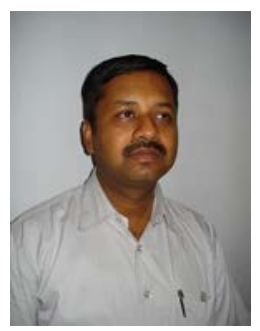

Dr. Himanshu Aggarwal Ph.D., is currently serving as Professor in Department of Computer Engineering at Punjabi University, Patiala. He has more than 22 years of teaching experience and served academic institutions such as Thapar Institute of Engineering \& Technology, Patiala, Guru Nanak Dev Engineering College, Ludhiana and Technical Teacher's Training Institute, Chandigarh. He is an active researcher who has supervised more than 30 M.Tech. Dissertations and contributed 80 articles in various Research Journals. He is guiding PhD to 8 scholars and 5 have completed their PhD. He is on the Editorial Board of 9 Journals and Review Boards of 5 Journals of repute. His areas of interest are Software Engineering, Computer Networks, Information Systems, ERP and Parallel Computing. Himanshu Aggarwal can be contacted at: himanshu.pup@gmail.com 